\title{
Subclinical hyperthyroidism is associated with increased risk of vertebral fractures in older men
}

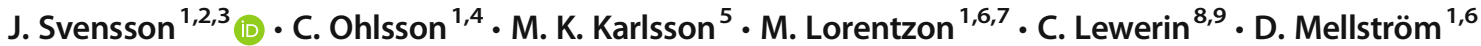

Received: 7 November 2020 / Accepted: 14 April 2021 / Published online: 19 May 2021

(C) The Author(s) 2021

\begin{abstract}
Summary In elderly men included in MrOS-Sweden, subclinical hyperthyroidism (SHyper) was markedly associated with increased risk of vertebral fractures.

Introduction Overt hyperthyroidism is associated with increased risk of fractures. However, only a few studies have investigated whether SHyper is associated with fracture risk in elderly men. We therefore investigated if SHyper was a risk factor for fractures in Swedish men.

Methods We followed (median 9.8 years) elderly men ( $n=1856$; mean age 75, range 69-81 years) participating in the Gothenburg and Malmö subcohorts of the prospective, population-based MrOS-Sweden study. The statistical analyses included Cox proportional hazards regression. SHyper was defined as serum thyroid-stimulating hormone (TSH) $<0.45 \mathrm{mIU} / \mathrm{L}(n=38)$. Results SHyper was associated with increased risk of all fractures $[n=456$; hazard ratio (HR) adjusted for age, study center, and levothyroxine treatment $=1.99,95 \%$ confidence interval $(\mathrm{CI}): 1.20-3.32]$, major osteoporotic fractures (MOF, $n=338 ; \mathrm{HR} 2.44$, 95\% CI: $1.42-4.21)$, and vertebral fractures $(n=176$; HR 3.79, 95\% CI: 2.02-7.11). These associations remained after full adjustment for covariates including total hip bone mineral density and in subanalyses including only men with serum free thyroxine $\leq$ the upper normal limit. However, after exclusion of men receiving levothyroxine treatment, the associations with all fractures and MOF lost significance.

Conclusions In elderly Swedish men, there was a strong association between SHyper and increased risk of vertebral fractures, whereas the associations with all incident fractures and MOF need to be confirmed in further studies.
\end{abstract}

Keywords Fracture risk · Older men · Serum thyroid-stimulating hormone $\cdot$ Subclinical hyperthyroidism $\cdot$ Vertebral fracture

\section{Introduction}

Thyroid hormones affect the function of most tissues. In the skeleton, they increase bone turnover, resulting in decreased

J. Svensson

johan.svensson@medic.gu.se

1 Center for Bone and Arthritis Research, Department of Internal Medicine and Clinical Nutrition, Institute of Medicine, Sahlgrenska Academy, University of Gothenburg, Gothenburg, Sweden

2 Department of Internal Medicine, Region Västra Götaland, Skaraborg Central Hospital, Skövde, Sweden

3 Department of Internal Medicine, Sahlgrenska University Hospital, Gröna Stråket 8, SE-413 45 Gothenburg, Sweden

4 Department of Drug Treatment, Region Västra Götaland, Sahlgrenska University Hospital, Gothenburg, Sweden bone mass if thyroid hormone activity is high [1]. This decrease is essentially reversible, providing that lasting structural damage has not been induced due to trabecular perforations [2]. In children, severe hypothyroidism leads to reduced

5 Clinical and Molecular Osteoporosis Research Unit, Department of Clinical Sciences and Orthopedics, Lund University, Malmö, Sweden

6 Geriatric Medicine, Department of Internal Medicine and Clinical Nutrition, Institute of Medicine, Sahlgrenska Academy, University of Gothenburg, Gothenburg, Sweden

7 Mary McKillop Institute for Health Research, Australian Catholic University, Melbourne, Australia

8 Department of Hematology and Coagulation, Region Västra Götaland, Sahlgrenska University Hospital, Gothenburg, Sweden

9 Department of Internal Medicine and Clinical Nutrition, Institute of Medicine, Sahlgrenska Academy, University of Gothenburg, Gothenburg, Sweden 
growth and delayed bone maturation [1], whereas overt hyperthyroidism causes short stature due to premature bone maturation [1]. In adult life, untreated overt hyperthyroidism is an established cause of secondary osteoporosis and associated with increased risk of fractures [1]. Moreover, overtreatment with levothyroxine results in thyroid-stimulating hormone (TSH) suppression, reduced bone mineral density (BMD), and increased risk of fractures $[1,3,4]$.

Not only overt hyperthyroidism, but also subclinical hyperthyroidism (SHyper), in which serum TSH levels are low in the presence of free triiodothyronine (T3) and free thyroxine (T4) levels within the normal range, is associated with reduced bone health [1]. Although the results are not fully consistent [1], low-normal TSH values have generally been associated with decreased BMD [5, 6] and modest increases in the risk of fractures $[5,7,8]$. In a pooled analysis of 5458 individuals from 6 prospective cohorts, SHyper was associated with loss of femur neck BMD, tended to be associated with loss of total hip BMD, but was not associated with loss of lumbar spine BMD [9]. Also in terms of the association between SHyper and fracture risk, individual studies have shown varying results [1]. In a meta-analysis by Blum et al., SHyper defined as $\mathrm{TSH}<0.45 \mathrm{mIU} / \mathrm{L}$ was associated with increased risk of any fracture and hip fracture, whereas there were only trends to associations with increased risks of spine and non-spine fractures [10]. However, the associations were stronger if TSH levels were less than $0.10 \mathrm{mIU} / \mathrm{L}$ and in endogenous SHyper [10]. In another meta-analysis by Zhu et al., in which the cutoff TSH value for SHyper varied between 0.30 and $0.55 \mathrm{mIU} /$ $\mathrm{L}$, there were significant but moderate associations between SHyper and increased risk of any, hip, spine, and non-spine fractures [11].

Most previous studies in SHyper have been performed in postmenopausal women $[5,12,13]$. In study populations consisting of both genders, the association between SHyper in men and hip fracture risk has not been consistent [14-17]. In two prospective studies performed only in men (total cohorts, $n=1817$ and $n=3338$, respectively), there was no association between SHyper and increased risk of hip fractures $[18,19]$ or non-spine fractures [18]. However, in the MrOS-US study, reduced serum TSH (per standard deviation decrease) was associated with increased risk of hip fracture in the total cohort [18]. Furthermore, none of these two studies evaluated the association between SHyper in older men and the risk of vertebral fractures.

In summary, the results of two meta-analyses suggest that SHyper is associated with increased risk of all fractures [10, 11]. However, most individual studies have been performed in postmenopausal women or in study populations consisting of both men and women, and to our knowledge, only two previous prospective studies have been performed in study populations consisting only of older men. In the present study of elderly men, we evaluated the association between SHyper and fracture risk in two well-controlled subcohorts of the prospective, population-based MrOS-Sweden study with access to validated fracture data based on computerized X-ray archives. We hypothesized that SHyper was associated with increased fracture risk.

\section{Materials and methods}

\section{Study population and ethics}

The Osteoporotic Fractures in Men Study (MrOS) is an international, multicenter study that includes older men. The present study involved the Malmö $(n=1005)$ and Gothenburg $(n=$ 1010) subcohorts of MrOS-Sweden. In MrOS-Sweden, men aged 69-81 years were randomly identified using national population registers, contacted, and asked to participate [20]. To be included in the study, the men had to be able to walk without assistance, sign an informed consent, and provide self-reported information. There were no other exclusion criteria. Among all the men contacted, $45 \%$ participated in the study. At baseline (years 2001 to 2004), the participants were physically examined, a questionnaire was answered, blood samples were drawn, and BMD was measured (see below). Then, during the follow-up, all new fractures were registered until the end of 2013.

In the present study, we investigated whether SHyper, defined as baseline serum TSH concentration $<0.45 \mathrm{mIU} / \mathrm{L}[10$, $21,22]$, was associated with the risk of all fractures, major osteoporotic fractures (MOF), vertebral fractures, or hip fractures. Measurements of serum TSH were available in 1856 of the elderly men ( $92 \%$ of the entire cohort).

All procedures were performed in accordance with the ethical standards of the institutional research committees and with the 1964 Helsinki Declaration and its later amendments. The study was approved by the ethics committees at Lund and Gothenburg Universities (LU 693/00 and Gbg M 014-1). Written informed consent was obtained from all participants.

\section{Assessment of BMD}

Areal BMD (aBMD; $\mathrm{g} / \mathrm{cm}^{2}$ ), later referred to as BMD, was assessed using dual-energy X-ray absorptiometry (DXA) with the Lunar Prodigy DXA (GE Lunar Corp., Madison, WI, USA) in Malmö and the Hologic QDR 4500/A-Delphi (Hologic, Waltham, MA, USA) in Gothenburg. Because the DXA measurements were performed with different equipment, all values for total hip BMD and lumbar spine BMD were standardized; the method used to calculate standardized BMD (sBMD) values has been described previously [20]. The coefficients of variation (CVs) for the BMD measurements varied from 0.5 to $3 \%$. 


\section{Assessment of covariates}

Body height and weight were measured using standard equipment. Body mass index (BMI) was calculated as weight (in kilograms) divided by height (in meters) squared. Appendicular lean mass was assessed using the Lunar Prodigy DXA (GE Lunar Corp.) in the Malmö cohort and the Hologic QDR 4500/A-Delphi (Hologic, Inc.) in the Gothenburg cohort. Hand grip strength was determined using a Jamar® hand dynamometer (Jackson, MI, USA) [23]. The maximum value (kilograms) from two trials from both hands was analyzed. Walking speed was calculated after determining the duration in seconds of a $6 \mathrm{~m}$ walk at normal pace. Information of smoking (yes, no, former) was obtained using a standardized questionnaire.

\section{Biochemical procedures}

Blood samples were drawn from all study participants and serum was separated, aliquoted, and stored at $-80{ }^{\circ} \mathrm{C}$ until analyses. Serum concentrations of TSH and free T4 were analyzed on the Roche Modular system (Roche Diagnostics Scandinavia AB, Solna, Sweden) as described previously [24]. The total CV of the TSH assay was $\leq 10 \%$ and the detection limit was $0.005 \mathrm{mIU} / \mathrm{L}$. The total $\mathrm{CV}$ of the free $\mathrm{T} 4$ assay was $\leq 10 \%$ and the detection limit was $1.5 \mathrm{pmol} / \mathrm{L}$.

\section{Assessment of fractures}

The participants were followed for 8.9 (SD 2.8) years on average (median 9.8 years, 25 th-75th percentiles: 7.5-10.8 years) after the baseline examination. The follow-up time was recorded from the date of baseline visit to the time of fracture or the date of death or the end of the study (December 31, 2013). Emigrants were followed up to the day of emigration. All fractures were recorded, and when subjects suffered from a first fracture at all sites during the followup, the follow-up time for respective fracture was analyzed. Deaths were documented from the National Cause of Death Register that includes all deaths in Sweden. All Swedish citizens have a unique personal registration number that enables access to information about the time as well as the site of fracture using computerized X-ray archives. The definition of a vertebral fracture was that the participant reported clinical symptoms after the baseline visit and that a vertebral fracture was confirmed by physician review of the radiology report. Thus, all fractures (vertebral and others) included in the analysis were confirmed by physician review of the radiology reports. Fractures reported by the study participants that were not possible to confirm by radiology were excluded. Further fracture analysis was then made of all validated fractures, vertebral fractures, hip fractures, and major osteoporotic fractures (MOF). MOF was defined as fracture of the hip, pelvis, proximal humerus, forearm, and vertebral fractures.

\section{Statistical analysis}

Statistical analyses were performed using SPSS for Windows (version 25.0, IBM Corp., Armonk, NY, USA). Unless otherwise stated, the descriptive statistical results at baseline are shown as the mean and standard deviation (SD). Betweengroup differences were examined using one-way analysis of variance (ANOVA) for continuous variables and using chisquare tests for categorical variables.

We used Cox proportional hazards regression to analyze the association between SHyper and the risk of all fractures, MOF, vertebral fractures, or hip fractures. Hazard ratios (HRs) and $95 \%$ confidence intervals (CIs) were calculated for the risk of having a fracture in men with SHyper compared to men with serum TSH $\geq 0.45 \mathrm{mIU} / \mathrm{L}$. We also performed similar Cox proportional regression analyses in men with SHyper and normal serum free T4 (exclusion of men with serum free $\mathrm{T} 4>$ the upper limit of the reference range of $22 \mathrm{pmol} / \mathrm{L}$ ), men with endogenous SHyper (exclusion of men receiving levothyroxine), and men with endogenous SHyper and normal serum free $\mathrm{T} 4$ (exclusion of men receiving levothyroxine as well as men with serum free T4 $>22 \mathrm{pmol} / \mathrm{L}$ ). Finally, in the total study population, we used Cox proportional hazards regression analyses to determine whether serum concentrations of TSH or free T4 as standardized continuous variables (per SD decrease in TSH and per SD increase in free T4) were associated with the risk of all fractures, MOF, vertebral fractures, or hip fractures. We adjusted all estimates for age, MrOS site, and treatment with levothyroxine (yes/no) (base model). Moreover, to examine the independent effect of SHyper on fracture risk, further adjustments were made for BMI, appendicular lean mass, grip strength, walking speed, and smoking status (model A). In model B, we also included total hip sBMD as a covariate. A two-sided $P<0.05$ was considered statistically significant.

\section{Results}

\section{Baseline characteristics}

Table 1 shows the baseline characteristics of the 38 elderly men with SHyper (serum TSH $<0.45 \mathrm{mIU} / \mathrm{L}$ ) and the 1818 men with serum TSH $\geq 0.45 \mathrm{mIU} / \mathrm{L}$. As expected, serum TSH was lower, and serum free T4 was higher, in the men with SHyper. Levothyroxine treatment was more common in the SHyper group. There were no between-group differences in age, BMI, appendicular lean mass, grip strength, walking speed, or lumbar spine L1 to L4 sBMD. The men with SHyper had lower total hip sBMD and higher prevalence of 
Table 1 Baseline characteristics of the study population

\begin{tabular}{|c|c|c|c|}
\hline Variable & $\begin{array}{l}\text { Subclinical } \\
\text { hyperthyroidism }^{\#} \\
n=38\end{array}$ & $\begin{array}{l}\text { Other men }^{\# \#} \\
n=1818\end{array}$ & $\begin{array}{l}P- \\
\text { value }\end{array}$ \\
\hline Serum TSH (mIU/L) & $0.21(0.15)$ & $2.65(3.63)$ & $<0.001$ \\
\hline Serum free $\mathrm{T} 4(\mathrm{pmol} / \mathrm{L})$ & $19.1(6.4)$ & $17.1(3.4)$ & $<0.001$ \\
\hline Levothyroxine treatment, $n(\%)$ & $9(23.7)$ & $22(1.2)$ & $<0.001$ \\
\hline Age (years) & $75.9(3.1)$ & $75.4(3.2)$ & 0.31 \\
\hline BMI $\left(\mathrm{kg} / \mathrm{m}^{2}\right)$ & $26.7(3.3)$ & $26.4(3.6)$ & 0.60 \\
\hline Appendicular lean mass (kg) & $25.1(3.6)$ & $24.5(3.3)$ & 0.33 \\
\hline Grip strength (kg) & $42.7(7.5)$ & $43.2(8.0)$ & 0.68 \\
\hline Walking speed $(\mathrm{m} / \mathrm{s})$ & $1.35(0.24)$ & $1.36(0.30)$ & 0.79 \\
\hline Current smoking (yes, no, former), $\%(n)$ & $21 / 18 / 61(8 / 7 / 23)$ & $\begin{array}{l}9 / 35 / 56 \\
\quad(164 / 625 / 1006)\end{array}$ & 0.01 \\
\hline Lumbar spine $\mathrm{L} 1$ to $\mathrm{L} 4 \mathrm{sBMD}\left(\mathrm{g} / \mathrm{cm}^{2}\right)$ & $1.08(0.16)$ & $1.13(0.19)$ & 0.17 \\
\hline Total hip sBMD $\left(\mathrm{g} / \mathrm{cm}^{2}\right)$ & $0.89(0.14)$ & $0.94(0.14)$ & $<0.05$ \\
\hline
\end{tabular}

If not otherwise stated, values are given as means (SD). Between-group differences were examined using one-way analysis of variance (ANOVA) for continuous variables and using chi-square tests for categorical variables

${ }^{*}$ Men with serum TSH $<0.45 \mathrm{mIU} / \mathrm{L}$

\#\# Men with serum TSH $\geq 0.45 \mathrm{mIU} / \mathrm{L}$

$B M I$, Body mass index; free T4, free thyroxine; $s B M D$, standardized bone mineral density; TSH, thyroidstimulating hormone smoking. One man in the SHyper group, who received levothyroxine treatment, had a previous history of thyroid cancer. None of the men with serum TSH $\geq 0.45 \mathrm{mIU} / \mathrm{L}$ had previous thyroid cancer. Exclusion of the man with previous thyroid cancer did not alter the results of the present study (data not shown).

\section{SHyper and risk of fractures}

During follow-up [mean 8.9 (SD 2.8) years, median 9.8 (25th-75th percentiles: $7.5-10.8)$ years], $456(25 \%)$ of the men suffered from a fracture. Analysis of risks in the men with SHyper vs. that in other men using Cox proportional hazards regression demonstrated that SHyper was associated with increased risks of all incident fractures (base model: HR 1.99, 95\% CI: 1.20 3.32), MOF (base model: HR 2.44, 95\% CI: 1.42-4.21), and vertebral fractures (base model: HR 3.79, 95\% CI: 2.02-7.11) (Table 2). All these associations remained significant after full adjustment for covariates (Table 2). However, the association between SHyper and the risk of hip fractures could not be evaluated in a meaningful way as there was only one incident hip fracture in the men with SHyper (data not shown). Finally, cumulative survival curves further illustrated that SHyper was associated with increased risk of all fractures (log-rank test, $P$ $<0.01$; data not shown), MOF $(P<0.001$; Fig. 1a), and vertebral fractures $(P<0.001$; Fig. $1 \mathrm{~b})$.
Table 2 Risk (hazard ratios and 95\% confidence intervals) of first fracture (all fractures, major osteoporotic fractures, or vertebral fractures) in subclinical hyperthyroidism

\begin{tabular}{lll}
\hline & Subclinical hyperthyroidism & Other men \\
\hline All fractures & & \\
Fractures, $n(\%)$ & $16(42.1 \%)$ & $440(24.2 \%)$ \\
Base model & $1.99(1.20-3.32)$ & 1.0 (referent) \\
Multivariate model A & $2.06(1.21-3.50)$ & 1.0 (referent) \\
Multivariate model B & $1.87(1.10-3.20)$ & 1.0 (referent) \\
Major osteoporotic fractures & & \\
Fractures, $n(\%)$ & $14(36.8 \%)$ & 324 (17.8\%) \\
Base model & $2.44(1.42-4.21)$ & 1.0 (referent) \\
Multivariate model A & $2.49(1.41-4.41)$ & 1.0 (referent) \\
Multivariate model B & $2.21(1.25-3.91)$ & 1.0 (referent) \\
Vertebral fractures & & 165 (9.1\%) \\
Fractures, $n(\%)$ & $11(28.9 \%)$ & 1.0 (referent) \\
Base model & $3.79(2.02-7.11)$ & 1.0 (referent) \\
Multivariate model A & $3.73(1.92-7.26)$ & 1.0 (referent) \\
Multivariate model B & $3.39(1.73-6.63)$ &
\end{tabular}

Hazard ratios were calculated using Cox proportional hazards regression

${ }^{\#}$ Men with serum TSH $<0.45 \mathrm{mIU} / \mathrm{L}$

${ }^{\# \#}$ Men with serum $\mathrm{TSH} \geq 0.45 \mathrm{mIU} / \mathrm{L}$

Base model: adjustment for age, MrOS site, and levothyroxine treatment (yes/no)

Multivariate model A: age, MrOS site, levothyroxine treatment, BMI, appendicular lean mass, grip strength, walking speed, and smoking status (yes, no, former)

Multivariate model B: age, MrOS site, levothyroxine treatment, BMI, appendicular lean mass, grip strength, walking speed, smoking status, and total hip sBMD 
Fig. 1 Subclinical hyperthyroidism is associated with increased fracture risk. Kaplan-Meier survival curves for a major osteoporotic fractures (MOF; log-rank test: $P<0.001$ ) and $\mathbf{b}$ vertebral fractures $(P<$ $0.001)$. Green, men with subclinical hyperthyroidism (serum TSH $<0.45 \mathrm{mIU} / \mathrm{L}$ ), blue, other men
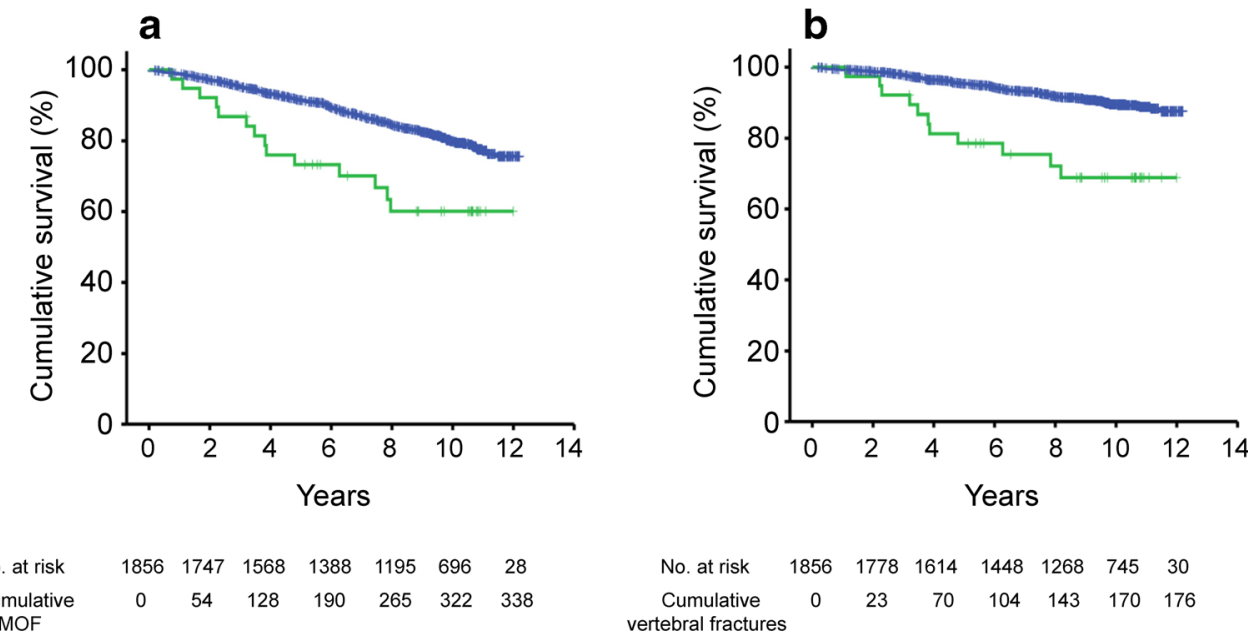

\section{Analyses in men with serum free T4 equal to or below the upper normal limit}

In subanalyses, we excluded men with serum free $\mathrm{T} 4>22$ $\mathrm{pmol} / \mathrm{L}$, thereby only analyzing men with serum free T4 equal to or below the normal upper limit of the reference range of the free T4 assay. In this group of men, using Cox proportional hazards regression analysis, SHyper $(n=29)$ was still associated with increased risk of all fractures [base model: HR 2.22, 95\% CI: 1.26-3.92; fully adjusted (model B): HR 1.90, 95\% CI: 1.04-3.47] and MOF [base model: HR 2.58, 95\% CI: 1.39-4.79; fully adjusted (model B): HR 2.07, 95\% CI: 1.08-3.98] compared to other men $(n=1776)$. Furthermore, also in this setting, there was a marked association between SHyper and increased risk of vertebral fractures (Table 3).

\section{Analyses in men not receiving levothyroxine}

We also performed subanalyses in which men with levothyroxine treatment were excluded. In the group of men with SHyper without levothyroxine treatment $(n=29)$, as compared to men not receiving levothyroxine $(n=1796)$, there was no association with the risk of all fractures [base model: HR 1.58, 95\% CI: 0.87-2.88; fully adjusted (model B): HR 1.47, 95\% CI: 0.78-2.78]. Furthermore, the association with $\mathrm{MOF}$ did not remain after additional adjustment for covariates (base model: HR 1.92, 95\% CI: 1.02-3.61; model A: HR 1.89, 95\% CI: 0.97-3.68; model B: HR 1.68, 95\% CI: 0.86-3.28). However, the association with vertebral fractures remained statistically significant in the Cox proportional hazards regression analyses (Table 3 ).

\section{Analyses in men with free $\mathrm{T} 4 \leq$ the upper normal limit and not receiving levothyroxine}

In further subanalyses, we excluded men with serum free T4> $22 \mathrm{pmol} / \mathrm{L}$ as well as men with levothyroxine treatment. In
Table 3 Risk (hazard ratios and 95\% confidence intervals) of first vertebral fracture in subclasses of subclinical hyperthyroidism (men with free $\mathrm{T} 4$ equal to or below the upper normal limit, men without levothyroxine treatment, and men with free $\mathrm{T} 4$ equal to or below the upper normal limit and without levothyroxine treatment)

\begin{tabular}{lcc}
\hline & $\begin{array}{l}\text { Subclinical } \\
\text { hyperthyroidism }\end{array}$ & Other men ${ }^{\#}$ \\
\hline Men with normal free T4\#\#\# & & \\
Vertebral fractures, $n$ (\%) & $9(31.0 \%)$ & 162 (9.1\%) \\
Base model & $4.02(2.00-8.10)$ & 1.0 (referent) \\
Multivariate model A & $3.68(1.75-7.75)$ & 1.0 (referent) \\
Multivariate model B & $3.28(1.54-6.98)$ & 1.0 (referent) \\
Men without levothyroxine treatment & \\
Vertebral fractures, $n$ (\%) & $9(31.0 \%)$ & 165 (9.2\%) \\
Base model & $3.42(1.74-6.71)$ & 1.0 (referent) \\
Multivariate model A & $3.31(1.61-6.80)$ & 1.0 (referent) \\
Multivariate model B & $3.05(1.48-6.26)$ & 1.0 (referent) \\
Men with normal free T4 ${ }^{\# \# \#}$ and without levothyroxine treatment \\
Vertebral fractures, $n$ (\%) & $7(31.8 \%)$ & 162 (9.2\%) \\
Base model & $3.55(1.65-7.60)$ & 1.0 (referent) \\
Multivariate model A & $3.12(1.37-7.12)$ & 1.0 (referent) \\
Multivariate model B & $2.83(1.24-6.48)$ & 1.0 (referent) \\
\hline
\end{tabular}

Hazard ratios were calculated using Cox proportional hazards regression ${ }^{\text {\# }}$ Men with serum TSH $<0.45 \mathrm{mIU} / \mathrm{L}$

\#\# Men with serum TSH $\geq 0.45 \mathrm{mIU} / \mathrm{L}$

\#\#\# Men with serum free T4 $\leq 22 \mathrm{pmol} / \mathrm{L}$ (serum free T4 equal to or below the normal upper limit of the reference range of the free T4 assay)

Base model: adjustment for age, MrOS site, and levothyroxine treatment (yes/no)

Multivariate model A: age, MrOS site, levothyroxine treatment, BMI, appendicular lean mass, grip strength, walking speed, and smoking status (yes/no/former)

Multivariate model B: age, MrOS site, levothyroxine treatment, BMI, appendicular lean mass, grip strength, walking speed, smoking status, and total hip sBMD 
these men, SHyper $(n=22)$ was not significantly associated with the risk of all fractures [base model: HR 1.79, 95\% CI: 0.92-3.48; fully adjusted (model B): HR 1.52, 95\% CI: $0.75-$ 3.09], and the significant association with MOF was lost after additional adjustment for covariates [base model: HR 2.09, 95\% CI: 1.03-4.24; model A: HR 1.86, 95\% CI: 0.87-3.96; model B: HR 1.62, 95\% CI: 0.76-3.46]. In contrast, the risk of vertebral fractures was still significantly increased in men with SHyper compared to that in other men $(n=1759)$ [HR 3.55, 95\% CI: 1.65-7.60; fully adjusted (model B): HR 2.83, 95\% CI: 1.24-6.48; Table 3].

\section{Analyses after excluding high serum TSH}

In final subanalyses, we excluded men with serum free T4 > $22 \mathrm{pmol} / \mathrm{L}$, men with levothyroxine treatment, and in addition men with serum TSH $\geq 4.5 \mathrm{mIU} / \mathrm{L}$. Thus, in these analyses, the men not having SHyper $(n=1623)$ were biochemically defined as euthyroid [10, 21, 22]. In the Cox proportional hazards regression analyses, SHyper $(n=22)$ was not associated with the risk of all fractures in any model (data not shown). Moreover, the significant association with MOF did not remain after additional adjustments for covariates (base model: HR 2.08, 95\% CI: 1.03-3.61; model A: HR 1.86. 95\% CI: 0.87-3.96; model B: HR 1.63, 95\% CI: 0.76-3.48). However, the risk of vertebral fractures [base model: HR 3.49, 95\% CI: 1.62-7.48; fully adjusted (model B): HR 2.92, 95\% CI: 1.28-6.67] was still significantly increased.

\section{Analysis in the total study population}

In the total study population ( $n=1856)$, we used Cox proportional hazards regression analyses to assess whether TSH or free T4 levels as standardized continuous variables were associated with the risk of fractures. Serum TSH (per SD decrease) was not associated with the risk of vertebral fractures [base model: HR 1.23, 95\% CI: 0.86-1.74; fully adjusted (model B): HR 1.12, 95\% CI: $0.82-1.52]$ or the risk of other fractures (data not shown). Serum free T4 (per SD increase) was not associated with the risk of all fractures, MOF, or vertebral fractures (data not shown). There was a significant association between serum free T4 (per SD increase) and hip fracture risk (base model: HR 1.14, 95\% CI: 1.004-1.30), which lost significance after additional adjustments (model A: HR 1.14, 95\% CI: 0.95-1.36; model B: HR 1.11, 95\% CI: $0.89-1.39$ ).

\section{Discussion}

The association between SHyper and fracture risk has been evaluated in postmenopausal women $[5,12,13]$ or in study populations consisting of both women and men [14-17], but there are only two previous prospective studies in study populations consisting only of older men $[18,19]$. In the present study, we evaluated the association between SHyper and fracture risk in two of the well-controlled MrOS-Sweden subcohorts with reliable fracture data based on computerized $\mathrm{X}$-ray archives. Our results demonstrate that SHyper in elderly men is associated with a robust and marked increase in the risk of vertebral fractures. SHyper was also associated with increased risk of all fractures and MOF. The latter associations remained after exclusion of men with serum free $\mathrm{T} 4$ above the reference range, but they lost significance after exclusion of men receiving levothyroxine treatment.

In the present study, $38(2.0 \%)$ of the totally 1856 men suffered from SHyper, which is in line with that found in some previous studies [10,25]. At baseline, measures of body composition and physical performance did not differ between the two study groups, whereas the men with SHyper had lower total hip SBMD and also non-significantly tended to have lower lumbar spine L1 to L4 sBMD compared to the men with serum TSH $\geq 0.45 \mathrm{mIU} / \mathrm{L}$. Although the results have varied between studies, our results to some extent converge with those of a pooled analysis of 6 prospective cohorts $(n=$ 5458). In the latter study, SHyper was associated with loss of femur neck BMD, tended to be associated with loss of total hip BMD, but was not associated with loss of lumbar spine BMD [9]. Furthermore, at baseline of the present study, smoking was more prevalent in the men with SHyper. However, in the Cox proportional hazards regression analyses, we adjusted for smoking status and several other covariates. In the final model (model B), we also adjusted for total hip sBMD although it could be argued that reduced BMD is a mediator, not a confounder, in terms of the association between SHyper and fracture risk.

A major finding of the present study is that SHyper was associated with a 3- to 4-fold increase in the risk of incident vertebral fractures. This association was only marginally attenuated by adjustment for covariates and remained statistically significant also after exclusion of men with elevated serum free T4 ( $>22 \mathrm{pmol} / \mathrm{L})$ and men receiving levothyroxine treatment. Moreover, the association also remained in the men with endogenous SHyper and normal serum free $\mathrm{T} 4(\mathrm{TSH}<0.45 \mathrm{mIU} /$ $\mathrm{L}$, free $\mathrm{T} 4 \leq 22 \mathrm{pmol} / \mathrm{L}$, and no levothyroxine) as compared to euthyroid men (serum TSH between $\geq 0.45 \mathrm{mIU} / \mathrm{L}$ and $<4.5$ $\mathrm{mIU} / \mathrm{L}$, free $\mathrm{T} 4 \leq 22 \mathrm{pmol} / \mathrm{L}$, and no levothyroxine). It could be hypothesized that we were able to detect a significant association between SHyper and increased risk of vertebral fractures because we had access to reliable fracture data based on computerized X-ray archives. Furthermore, our findings are in some agreement with the results of the meta-analysis by Blum et al. [10]. This meta-analysis demonstrated that SHyper was associated with a more marked risk of fractures in men than in women [10]. Although none of the individual studies included in the meta-analysis could detect any significant association between 
SHyper and spine fracture risk among men, the pooled results showed a significantly increased risk of spine fractures in men with SHyper $(n=282)$ [10].

To our knowledge, the present study is the third prospective study that has investigated the association between SHyper and fracture risk in a study population consisting only of older men. In the two previous studies (total cohorts, $n=$ 1817 and $n=3338$, respectively) $[18,19]$, the mean age at baseline (74 and 77 years, respectively) was approximately similar as that in the present study ( 75 years). None of the two earlier studies detected any association between SHyper and the risk of incident hip fractures [18, 19] or non-spine fractures [18], but in the total MrOS-US cohort, reduced serum TSH (per standard deviation decrease) was associated with increased risk of hip fracture [18]. Furthermore, as none of these two studies evaluated the association with the risk of vertebral fractures $[18,19]$, our finding of a strong and robust association between SHyper and incident vertebral fractures adds novel information. However, a limitation of our study as well as the two other studies $[18,19]$ is that there were very few incident hip fractures in the included men with SHyper.

In addition to the increased risk of vertebral fractures, we also observed that SHyper in men was associated with increased risk of all incident fractures and MOF. These associations remained statistically significant after full adjustment for covariates and after exclusion of men with serum free $\mathrm{T} 4$ above the reference range. However, when men receiving levothyroxine treatment were excluded, the associations lost significance after full adjustment for covariates. In earlier studies, the influence of levothyroxine treatment on fracture risk has been moderate [1], and of less importance than that exerted by endogenous SHyper [10]. Therefore, it seems less likely that levothyroxine treatment was a major confounder in terms of the associations between SHyper and the risks of all incident fractures and MOF. Instead, it could be hypothesized that the lack of statistical significance between SHyper and the risks of all fractures and MOF after exclusion of men receiving levothyroxine was a consequence of lack of sufficient statistical power, which could be supported by the fact that HRs remained relatively high. In additional support of this, in the meta-analysis by Blum et al. [10], SHyper was associated with increased risk of all fractures in men [10].

In our total study population, serum concentrations of TSH or free T4 as standardized continuous variables were not associated with the risk of fractures after adjustment for multiple covariates (models A and B). Thus, serum TSH was not associated with the risk of vertebral fractures in the total study population. This suggests that increased risk of vertebral fractures was specifically associated with SHyper in men. However, the mechanisms underlying this association are not fully clear. The association between SHyper and increased risk of vertebral fractures remained after full adjustment for covariates (model B), which included adjustment for variables such as BMI, appendicular lean mass, grip strength, walking speed, and total hip sBMD. However, SHyper could have induced alterations in bone turnover or musculoskeletal function that were not captured by the tests performed in our study.

In our study as in several previous studies [10,21, 22], a broader serum TSH range $(<0.45 \mathrm{mIU} / \mathrm{L})$ was used to define SHyper than that used in clinical practice, which could limit the clinical interpretations of our findings. However, even so, it is important that clinicians are aware of the increased risk of vertebral fractures in men with low serum TSH $(<0.45 \mathrm{mIU} /$ L). In a prospective study of low-risk differentiated thyroid cancer patients, suppressive doses of levothyroxine increased the risk of postoperative osteoporosis without beneficial effect on tumor recurrence [26]. Therefore, the focus of long-term levothyroxine treatment in low-risk thyroid cancer patients should be to avoid therapeutic harm $[1,26]$.

Our study has several strengths and weaknesses. Strengths are the relatively long follow-up and the reliable fracture data based on computerized X-ray archives, enabling us to include only X-ray-verified fractures with information about the time and site of each fracture. Furthermore, our cohort of elderly men was large and well-controlled, but even so, the subgroup with SHyper was small $(n=38)$. This likely resulted in limited statistical power, especially in terms of subanalyses, and we could not perform meaningful statistical analyses regarding the risk of hip fractures in men with SHyper. Levothyroxine treatment was recorded, and free $\mathrm{T} 4$ was measured, but except for previous history of thyroid cancer, it was not registered whether the men had a previous thyroid disorder. One additional limitation is that self-reported questionnaires were used, and we cannot exclude the possibility that this could have resulted in an underestimation of the prevalence of smoking. Finally, most measurements were only performed at baseline (thyroid hormones, sBMD, presence of levothyroxine treatment, and covariates/confounders), which could result in an underestimation of the true associations.

In summary, this is the third prospective study that has determined the association between SHyper and the risk of incident fractures in a study population consisting only of older men. We extend the previous knowledge by showing a marked and robust association between SHyper and increased risk of vertebral fractures in elderly men. SHyper was associated with increased risk of all incident fractures and MOF also after exclusion of men with serum free $\mathrm{T} 4$ above the reference range, but after exclusion of men receiving levothyroxine treatment, these associations lost statistical significance. A limitation of the present study is that we did not have sufficient statistical power to investigate the association between SHyper and the risk of hip fractures. Further studies are therefore needed in larger study populations to investigate the association between SHyper and the risk of incident fractures in more detail as well as whether treatment of SHyper can reduce the risk of vertebral fractures in older men. 
Acknowledgements We thank the MrOS study personnel for excellent research assistance.

Funding Open access funding provided by University of Gothenburg. This work was supported by the Swedish state under the agreement between the Swedish government and the county councils, the ALFagreement (ALFGBG-722371 and ALFGBG-437971).

Availability of data and material The raw data used in the current study are restricted in order to protect participant privacy, as required by data protection acts in Sweden. Data can be made accessible by request for researchers after permission from the Swedish Ethics Review Authority.

\section{Code availability Not applicable.}

\section{Declarations}

Ethics approval and consent to participate All procedures were performed in accordance with the ethical standards of the institutional research committees and with the 1964 Helsinki Declaration and its later amendments. The study was approved by the ethics committees at Lund and Gothenburg Universities (LU 693/00 and Gbg M 014-1). Written informed consent was obtained from all participants.

\section{Consent for publication Not applicable.}

\section{Conflicts of interest None.}

Open Access This article is licensed under a Creative Commons Attribution-NonCommercial 4.0 International License, which permits any non-commercial use, sharing, adaptation, distribution and reproduction in any medium or format, as long as you give appropriate credit to the original author(s) and the source, provide a link to the Creative Commons licence, and indicate if changes were made. The images or other third party material in this article are included in the article's Creative Commons licence, unless indicated otherwise in a credit line to the material. If material is not included in the article's Creative Commons licence and your intended use is not permitted by statutory regulation or exceeds the permitted use, you will need to obtain permission directly from the copyright holder. To view a copy of this licence, visit http:// creativecommons.org/licenses/by-nc/4.0/.

\section{References}

1. Bassett J, Williams G (2016) Role of thyroid hormones in skeletal development and bone maintenance. Endocr Rev 37:135-187

2. Mosekilde L, Eriksen E, Charles P (1990) Effects of thyroid hormones on bone and mineral metabolism. Endocrinol Metab Clin N Am 19:35-63

3. Abrahamsen B, Jørgensen H, Laulund A, Nybo M, Bauer D, Brix T, Hegedüs L (2015) The excess risk of major osteoporotic fractures in hypothyroidism is driven by cumulative hyperthyroid as opposed to hypothyroid time: an observational register-based time-resolved cohort analysis. J Bone Miner Res 30:898-905

4. Flynn R, Bonellie S, Jung R, MacDonald T, Morris A, Leese G (2010) Serum thyroid-stimulating hormone concentration and morbidity from cardiovascular disease and fractures in patients on longterm thyroxine therapy. J Clin Endocrinol Metab 95:186-193

5. Murphy E, Glüer C, Reid D, Felsenberg D, Roux C, Eastell R, Williams G (2010) Thyroid function within the upper normal range is associated with reduced bone mineral density and an increased risk of nonvertebral fractures in healthy euthyroid postmenopausal women. J Clin Endocrinol Metab 95:3173-3181

6. Roef G, Lapauw B, Goemaere S, Zmierczak H, Fiers T, Kaufman J, Taes Y (2011) Thyroid hormone status within the physiological range affects bone mass and density in healthy men at the age of peak bone mass. Eur J Endocrinol 164:1027-1034

7. Abrahamsen B, Jørgensen H, Laulund A, Nybo M, Brix T, Hegedüs L (2014) Low serum thyrotropin level and duration of suppression as a predictor of major osteoporotic fractures-the OPENTHYRO register cohort. J Bone Miner Res 29:2040-2050

8. Taylor P, Razvi S, Pearce S, Dayan C (2013) Clinical review: a review of the clinical consequences of variation in thyroid function within the reference range. J Clin Endocrinol Metab 98:3562-3571

9. Segna D, Bauer D, Feller M, Schneider C, Fink H, Aubert C, Collet T, da Costa B, Fischer K, Peeters R, Cappola A, Blum M, van Dorland H, Robbins J, Naylor K, Eastell R, Uitterlinden A, Rivadeneira Ramirez F, Gogakos A, Gussekloo J, Williams G, Schwartz A, Cauley J, Aujesky D, Bischoff-Ferrari H, Rodondi N, Thyroid Studies Collaboration (2018) Association between subclinical thyroid dysfunction and change in bone mineral density in prospective cohorts. J Intern Med 283:56-72

10. Blum M, Bauer D, Collet T, Fink H, Cappola A, da Costa B, Wirth C, Peeters R, Åsvold B, den Elzen W, Luben R, Imaizumi M, Bremner A, Gogakos A, Eastell R, Kearney P, Strotmeyer E, Wallace E, Hoff M, Ceresini G, Rivadeneira F, Uitterlinden A, Stott D, Westendorp R, Khaw K, Langhammer A, Ferrucci L, Gussekloo J, Williams G, Walsh J, Jüni P, Aujesky D, Rodondi N, Thyroid Studies Collaboration (2015) Subclinical thyroid dysfunction and fracture risk: a meta-analysis. JAMA 313:2055-2065

11. Zhu H, Zhang J, Wang J, Zhao X, Gu M (2019) Association of subclinical thyroid dysfunction with bone mineral density and fracture: a meta-analysis of prospective cohort studies. Endocrine E67: 685-698

12. Finigan J, Greenfield D, Blumsohn A, Hannon R, Peel N, Jiang G, Eastell R (2008) Risk factors for vertebral and nonvertebral fracture over 10 years: a population-based study in women. J Bone Miner Res 23:75-85

13. Bauer D, Ettinger B, Nevitt M, Stone K, Study of Osteoporotic Fractures Research Group (2001) Risk for fracture in women with low serum levels of thyroid-stimulating hormone. Ann Intern Med 134:561-568

14. Garin M, Arnold A, Lee J, Robbins J, Cappola A (2014) Subclinical thyroid dysfunction and hip fracture and bone mineral density in older adults: the cardiovascular health study. J Clin Endocrinol Metab 99:2657-2664

15. Lee J, Buzková P, Fink H, Vu J, Carbone L, Chen Z, Cauley J, Bauer D, Cappola A, Robbins J (2010) Subclinical thyroid dysfunction and incident hip fracture in older adults. Arch Intern Med 170: 1876-1883

16. Svare A, Nilsen T, Asvold B, Forsmo S, Schei B, Bjøro T, Langhammer A (2013) Does thyroid function influence fracture risk? Prospective data from the HUNT2 study, Norway. Eur J Endocrinol 169:845-852

17. Vadiveloo T, Donnan P, Cochrane L, Leese G (2011) The Thyroid Epidemiology, Audit, and Research Study (TEARS): morbidity in patients with endogenous subclinical hyperthyroidism. J Clin Endocrinol Metab 96:1344-1351

18. Waring A, Harrison S, Fink H, Samuels M, Cawthon P, Zmuda J, Orwoll E, Bauer D, Osteoporotic Fractures in Men (MrOS) Study (2013) A prospective study of thyroid function, bone loss, and fractures in older men: the MrOS study. J Bone Miner Res 28: $472-479$

19. Siru R, Alfonso H, Chubb S, Golledge J, Flicker L, Yeap B (2018) Subclinical thyroid dysfunction and circulating thyroid hormones 
are not associated with bone turnover markers or incident hip fracture in older men. Clin Endocrinol 89:93-99

20. Mellstrom D, Johnell O, Ljunggren O, Eriksson AL, Lorentzon M, Mallmin H, Holmberg A, Redlund-Johnell I, Orwoll E, Ohlsson C (2006) Free testosterone is an independent predictor of BMD and prevalent fractures in elderly men: MrOS Sweden. J Bone Miner Res 21:529-535

21. Aubert C, Floriani C, Bauer D, da Costa B, Segna D, Blum M, Collet T, Fink H, Cappola A, Syrogiannouli L, Peeters R, Åsvold B, den Elzen W, Luben R, Bremner A, Gogakos A, Eastell R, Kearney P, Hoff M, Le Blanc E, Ceresini G, Rivadeneira F, Uitterlinden A, Khaw K, Langhammer A, Stott D, Westendorp R, Ferrucci L, Williams G, Gussekloo J, Walsh J, Aujesky D, Rodondi N, Thyroid Studies Collaboration (2017) Thyroid function tests in the reference range and fracture: individual participant analysis of prospective cohorts. J Clin Endocrinol Metab 102:2719-2728

22. Floriani C, Feller M, Aubert C, M'Rabet-Bensalah K, Collet T, den Elzen W, Bauer D, Angelillo-Scherrer A, Aujesky D, Rodondi N (2018) Thyroid dysfunction and anemia: a prospective cohort study and a systematic review. Thyroid 28:575-582

23. Karlsson M, Ribom E, Nilsson J, Ljunggren Ö, Ohlsson C, Mellström D, Lorentzon M, Mallmin H, Stefanick M, Lapidus J,
Leung P, Kwok A, Barrett-Connor E, Orwoll E, Rosengren B (2012) Inferior physical performance tests in 10,998 men in the MrOS study is associated with recurrent falls. Age Ageing 41: 740-746

24. Lewerin C, Nilsson-Ehle H, Jacobsson S, Johansson H, Sundh V, Karlsson M, Lorentzon M, Barrett-Connor E, Vandenput L, Ohlsson C, Mellström D (2014) Serum estradiol associates with blood hemoglobin in elderly men: the MrOS Sweden study. J Clin Endocrinol Metab 99:2549-2556

25. Boekholdt S, Titan S, Wiersinga W, Chatterjee K, Basart D, Luben R, Wareham N, Khaw K (2010) Initial thyroid status and cardiovascular risk factors: the EPIC-Norfolk prospective population study. Clin Endocrinol 72:404-410

26. Wang L, Smith A, Palmer F, Tuttle R, Mahrous A, Nixon I, Patel S, Ganly I, Fagin J, Boucai L (2015) Thyrotropin suppression increases the risk of osteoporosis without decreasing recurrence in ATA low- and intermediate-risk patients with differentiated thyroid carcinoma. Thyroid 25:300-307

Publisher's note Springer Nature remains neutral with regard to jurisdictional claims in published maps and institutional affiliations. 